\title{
Primary Angiosarcoma of the Tongue: A Case Report
}

\author{
Vineet Chadha, Digvijay Singh Rawat, Beni Prasad \\ Department of Otorhinolaryngology, SMS Medical College, Jaipur, India \\ Email: chadhavineet2001@yahoo.in
}

Received June 21, 2012; revised July 31, 2012; accepted August 20, 2012

\begin{abstract}
Sarcomas are rare tumors of head and neck region and constitute less than $1 \%$ of all cancers in this region. Primary angiosarcoma of the oral cavity is an extremely rare malignancy with few reported cases in literature till now. We present a case of a 50-year-old woman who was diagnosed as angiosarcoma after appropriate histopathological and immunohistochemical evaluation.
\end{abstract}

Keywords: Angiosarcoma; Tongue Tumor; Immunohistochemistry

\section{Introduction}

Angiosarcoma is a malignant tumor of vascular endothelial cell origin with a definitively aggressive, malignant clinical course and a histopathologic appearance which is more atypical than the low-grade lesion called hemangioendothelioma. It is malignant counterpart of the hemangioma and one of the rarest among the various soft tissue sarcomas, comprising less than $1 \%$ of all sarcomas [1]. It is also known as malignant hemangioendothelioma, angioblastoma, hemangiosarcoma, and intravascular endothelioma. The exact etiology of angiosarcoma is unknown but trauma, radiation and angectesia have been linked with its etiology [2].

Angiosarcoma occurs most commonly in the skin and superficial soft tissues. Angiosarcoma of the head and neck is very rare and when it does occur in this region, the scalp and facial skin are the most common sites of origin [3]. Less than $8 \%$ of head and neck lesions arise from oral cavity sites Most cases have involved the mandible, but other less frequent sites of involvement have included the maxilla, parotid glands, lips, tongue, floor of mouth, cheek, palate and antrum.

Angiosarcoma of the oral region is a disease of older individuals, averaging more than 65 years of age, but it has also been reported in infancy. There is no gender predilection and the tumor is typically a solitary or multifocal submucosal nodule which may be bosselated, may be ulcerated, and may bleed spontaneously.

\section{Case Report}

A 50-year-old woman presented with 1-month history of swelling right lateral margin of tongue which was gradually progressive in size. She also complained of occasional episodes of bleeding, usually following meals. The mass was purplish in colour was around $4 \mathrm{~cm}$ in length along the lateral border, had a lobulated surface and was soft on palpation. Examination of nose, oropharynx and larynx was normal. No cervical lymphadenopathy was present and chest $\mathrm{X}$ ray was normal. She had a history of similar smaller lesion near the tip of the tongue 6 months back which was enucleated at another centre and its histopathology was a capillary hemangioma.

A wide local excision of the lesion was performed under general anaesthesia and the resected specimen was marked and sent for histopathological evaluation. The postoperative period was uneventful. The histopathology showed a malignant neoplasm showing vascular channels lined by enlarged endothelial cells and solid sheets of cells penetrating between collagen bundles and muscular tissue with moderate nuclear atypia and mitotic activity, consistent with angiosarcoma (Figure 1). Immunohistochemical marker study was performed which resulted in positive staining for factor VIII antigens, CD31 and thrombomodulin confirming the diagnosis of angiosarcoma. The patient currently remains asymptomatic without clinical or radiological evidence of any loco-regional recurrence and distant metastasis.

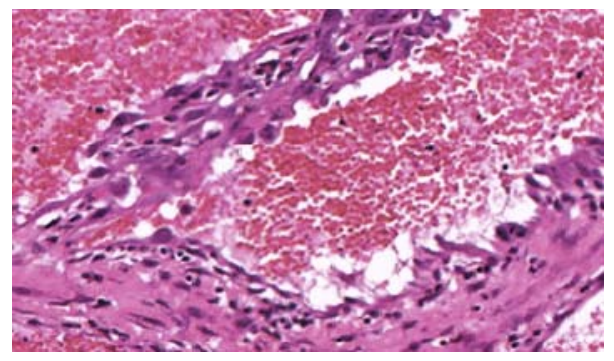

Figure 1. HPE showing irregularly shaped vascular spaces separated by collagenous stroma with mitotic figures. 


\section{Discussion}

Sarcomas are rarely found on the tongue and usually represent less than $1 \%$ of malignant tumors at this location [4]. Angiosarcoma is a xeroderma pigmentosum related tumor that usually arises in sun-exposed skin but can also arise in the oral cavity [5]. Angiosarcoma is one of the rarest tumors arising in the tongue, accounting for vanishingly small proportion of primary tongue sarcomas. The earliest reported case of angiosarcoma of tongue was in 1927 by J. H. Harter [6]. A review published by Fanburg-Smith et al. in 2003 illustrated that there were only 13 reported cases of primary angiosarcoma in English literature ( 9 by them and 4 by others) till then [7]. On search of Indian literature we found a single reported case of primary hemangiosarcoma of tongue by Rao in 1985 [8]. The present case is thus amongst one of the few reported cases of angiosarcoma in India. Because of its rarity, it practically is never considered in differential diagnosis of malignant lesions in this location.

Immunohistochemical analysis is essential for differentiating angiosarcoma from similar lesions in this location such as hemangioendothelioma, hemangiopericytoma, epithelioid sarcoma, Kaposi's sarcoma. Positive staining for factor VII and Ulex europaeus agglutinin has been used to diagnose a vascular pathology [7]. CD31, a membrane glycoprotein, is the best vascular marker for endothelial phenotype, particularly in poorly differentiated angiosarcoma, when Factor VIII and Ulex europaeus fail $[6,9]$. Positive staining for thrombomodulin and E-selectin also support the diagnosis of angiosarcoma [10].

Angiosarcoma usually spreads by hematogenous route and pulmonary metastases can occur. Lymphatic spread is reported in less than $20 \%$ of angiosarcomas in head and neck region. In our case no lymph node or distant metastasis were observed at initial presentation or during follow up period.

Surgery remains the main stay of therapy. A combined modality of treatment using postoperative radiotherapy may be used. The general outcome of primary angiosarcoma of oral cavity is good compared to primary cutaneous and deep soft tissue tumors. The mean survival period of 7.6 years has been documented for primary angiosarcomas. The patient was treated by wide local excision and has been kept under strict follow-up with regular clinical and radiological examination for local recurrence and pulmonary metastasis.

\section{Conclusion}

Angiosarcoma is a one of the rarest tumor to involve the tongue and whenever suspected should be dealt with care, using available immunohistochemical studies and vigilant follow-up.

\section{REFERENCES}

[1] N. B. Solomons and M. P. Stearns, "Haemangiosarcoma of the Maxillary Antrum," The Journal of Laryngology and Otology, Vol. 104, No. 10, 1990, pp. 831-834. doi:10.1017/S0022215100114045

[2] W. R. Panje, W. J. Morran, D. G. Bostwick and V. V. Kitt, "Angiosarcoma of the Head and Neck: Review of 11 Cases,” Laryngoscope, Vol. 96, No. 12, 1986, pp. 13811384.

[3] G. K. Maheshwari, H. Baboo, M. H. Patel, U. Gopal and K. Wadhwa, "Primary Angiosarcoma of the Larynx," Turkish Journal of Cancer, Vol. 34, No. 4, 2004, pp. 186188.

[4] J. C. Watkinson, J. A. Wilson and M. N. Gaze, "Tumors of Lip and Oral Cavity,” In: Stell and Maran's Head and Neck Surgery, 4th Edition, Hodder Arnold, London, 2000.

[5] M. T. Olson, K. B. Puttgen and W. H. Westra, "Angiosarcoma Arising from the Tongue of an 11-year-old Girl with Xeroderma Pigmentosum," Head and Neck Pathology, Vol. 6, No. 2, 2012, pp. 255-257. doi:10.1007/s12105-011-0303-X

[6] J. H. Harter, “Angiosarcoma of Tongue-Case Report," Laryngoscope, Vol. 37, No. 12, 1927, pp. 869-871. doi:10.1288/00005537-192712000-00003

[7] J. C. Fanburg-Smith, M. A. Furlong and E. L. Childers, "Oral and Salivary Gland Angiosarcoma: A Clinicopathologic Study of 29 Cases,” Modern Pathology, Vol. 16, No. 3, 2003, pp. 263-271. doi:10.1097/01.MP.0000056986.08999.FD

[8] K. K. Rao, H. N. Kumar and R. V. Rao, "Primary Haemangiosarcoma of the Tongue," International Journal of Oral and Maxillofacial Surgery, Vol. 15, No. 4, 1986, pp. 489-491. doi:10.1016/S0300-9785(86)80044-8

[9] S. S. Mullick, D. R. Mody and M. R. Schwartz, "Angiosarcoma at Unusual Sites: A Report of Two Cases with Aspiration Cytology and Diagnostic Pitfalls,” Acta Cytologica, Vol. 41, No. 3, 1997, pp. 839-844. doi:10.1159/000332713

[10] M. Tabata, K. Sugihara, R. Matsui, S. Yonezawa, K. Abeyama and I. Maruyama, "Angiosarcoma of the Tongue: Report of a Case with Immunohistochemical Findings," Journal of Oral Pathology \& Medicine, Vol. 28, No. 2, 1999, pp. 92-95. doi:10.1111/j.1600-0714.1999.tb02003.x 